\title{
Calcium sensitive receptor and claudin-14 expression in kidney tissues of two kidney stone models
}

\author{
Biao Qian ${ }^{1}$, Jihong Liu', Jingshen Wang' ${ }^{2}$ Zhiqiang $\mathrm{Hao}^{3}$, Qinzhang Wang
}

\author{
${ }^{1}$ Department of Urology, Tongji Hospital, Tongji Medical College, Huazhong University \\ of Science and Technology, Wuhan, Hubei, China \\ ${ }^{2}$ Department of Urology Surgery, the First Affiliated Hospital of Medical College in \\ Shihezi University, Shihezi, Xinjiang, China \\ ${ }^{3}$ Department of Urology, the First Affiliated Hospital of Medical College in Shihezi \\ University, Shihezi, Xinjiang, China
}

Submitted: 16 October 2018, accepted: 29 January 2019

Online publication: 19 March 2021

Arch Med Sci 2022; 18 (1): 251-256

DOI: https://doi.org/10.5114/aoms/103453

Copyright @ 2021 Termedia \& Banach

\begin{abstract}
A bstract
Intoduction: The differences in protein expression of calcium sensitive receptor (CaSR) and claudin-14 in a kidney stone model established by nanobacteria (NB) and ethylene glycol (EG) were compared.

Material and methods: Ninety Wistar male rats were randomly divided into the NB group, the EG group, and the blank control group (NC group), with 30 rats in each group. Three rats of each group were sacrificed every week after injection. Histopathology was used to evaluate the stone formation of each group. The expression of CaSR and claudin-14 protein was detected by immunohistochemistry every week.

Results: There was formation of bright crystals in the kidneys of the EG group and the NB group, but not the NC group. At the 3rd week, the expression of CaSR and claudin-14 in the kidney tissue of the EG group began to increase while that in the NB group increased at the $4^{\text {th }}$ week. The expression of CaSR and claudin-14 protein in the EG group was stronger than that in the NB group. Meanwhile, CaSR was expressed in the NC group but did not change significantly. Claudin-14 was not expressed in the NC group.

Conclusions: Our results indicate that the traditional EG kidney stone modeling method is more rapid than the NB kidney stone modeling method, with a high stone formation rate, and the CaSR and claudin-14 protein expression levels are higher. Meanwhile, the NB used to establish the kidney stone model was isolated from patients with kidney stones, which may imitate the process of natural formation of kidney stones of patients. Therefore, the results of our research are more conducive to related research on the etiology of stones.
\end{abstract}

Key words: calcium sensitive receptor, claudin-14, ethylene glycol, kidney stones, nanobacteria.

\section{Introduction}

Kidney stones are common diseases of the urinary system. Patients with stones represent the most numerous group in the emergency department, the inpatient department and the urology surgery department of general hospitals. In recent years, with the improvement of surgical methods, surgical methods have gradually become minimally invasive, and the rate of stone cure has increased, but the rate of stone recurrence

\author{
Corresponding author: \\ Jihong Liu's \\ Department of Urology \\ Tongji Hospital \\ Tongji Medical College \\ Huazhong University \\ of Science and Technology \\ Wuhan \\ 430030 Hubei, China \\ E-mail: jhliu@tjh.tjmu.edu.cn
}


remains high [1, 2]. Therefore, the mechanism of stone formation is an important breakthrough point for treatment of kidney stones. In recent years, it has been found that the calcium-sensing receptor (CaSR)-claudin-14 pathway is closely related to renal calcium reabsorption and stone formation [3]. CaSR is a G-protein coupled receptor that is significantly associated with urinary calcium excretion, which can be up-regulated by the thick ascending limb (TAL) tight junction protein claudin-14. Expression of claudin-14 inhibits renal tubular reabsorption of calcium ions [4].

In this study, the expression levels of CaSR and claudin-14 in two kidney stone models were observed and compared by establishing kidney stone models with ethylene glycol (EG) method and nano-bacteria (NB) method.

\section{Material and methods}

\section{Animals}

Ninety male SPF Wistar rats aged 6 weeks and weighing $200 \pm 20$ g were purchased from the Experimental Animal Center of Xinjiang Medical University (certificate No. SCXK (new) 2013 - 0001). Rats were randomly divided into the blank control group (NC group), the NB group and the EG group, with 30 rats in each group.

\section{Source of NB}

Morning urine samples were collected from patients with upper urinary calculi $(5 \mathrm{ml}$ each). After dilution 2 times with normal saline, filtration and centrifugation, NB solution was collected. Then, DMEM and heat inactivated fetal bovine serum were added and incubated at $5 \% \mathrm{CO}_{2}, 37^{\circ} \mathrm{C}$ for 28-30 days, and the solution was changed every 30 days. Non-contaminated specimens were chosen to make a NB suspension.

\section{Traditional glycol reagent configuration}

Preparation of EG solution: $10 \mathrm{ml}$ of EG (analytical grade) was weighed in a $100 \mathrm{ml}$ measuring cylinder, and the volume was adjusted to $1000 \mathrm{ml}$ with tap water to obtain a $1 \%$ EG solution. Preparation of ammonium chloride solution: $2 \mathrm{~g}$ of ammonium chloride crystal (analytical grade) was weighed with an electronic balance, and $1000 \mathrm{ml}$ of sterile double - distilled water was weighed in a measuring cylinder. The two were thoroughly mixed, and then a $2 \%$ ammonium chloride solution was obtained.

\section{Establishment of animal models}

Rats in the NC group were injected with $1.2 \mathrm{ml}$ of normal saline and fed with normal saline. Rats in the NB group received a tail vein injection of NB suspension (absorbance 1.00, $1.2 \mathrm{ml}$ ) and ga-vaged with normal saline. The rats in the EG group received $1 \%$ ethylene glycol drinking water and were given $2 \mathrm{ml}$ of $2 \%$ ammonium chloride by gavage. The entire modeling process lasts for 2 weeks.

\section{Specimen collection}

Rats were anesthetized with $10 \%$ chloral hydrate (i.p.) at a dose of $4 \mathrm{ml} / \mathrm{kg}$. After the muscles were relaxed, the abdomen was opened, and the bilateral kidney tissues were collected. After washing, some kidneys were quickly frozen and preserved. Approximately $0.5 \mathrm{~g}$ of the tissue near the coronal section of the kidney was picked with a thick needle. After drying, an infrared spectrum automatic analyzer was used to analyze the stone composition. Some kidneys were fixed with formaldehyde solution.

\section{Pathological examination}

The morphology and formation of the crystals of the stones were observed using a dissecting microscope, and the color, shape and size of the kidneys were observed with the naked eye. The fixed kidney specimens were quickly embedded, and $5 \mu \mathrm{m}$ thick transverse sections were prepared. Routine HE staining was performed. The formation of crystals and renal pathological changes were observed under an optical microscope. The kidney crystals were classified according to the following criteria [5]: Grade 0 indicates a negative result, and grades I to IV indicate positive results. If the bilateral kidneys showed a positive result at the same time, the crystals of only one kidney were counted.

\section{Immunohistochemical detection of CaSR and claudin-14 protein expression}

Immunohistochemistry was performed using the SP method. After paraffin embedding, the tissues were cut into $4 \mu \mathrm{m}$ sections. After routine dewaxing, sections were treated with $\mathrm{H}_{2} \mathrm{O}_{2}$ to remove endogenous peroxidase. After antigen retrieval and blocking, CaSR antibody (1 : 150) and claudin-14 antibody $(1: 200)$ were added and incubated overnight at $4^{\circ} \mathrm{C}$. After washing, the secondary antibody of horseradish-labeled rabbit anti-goat IgG was added and incubated. DAB was used for color development. After counterstaining with hematoxylin, the sections were dehydrated with different concentrations of ethanol, rendered transparent with xylene and mounted with neutral gum. The immunohistochemical staining of CaSR and claudin-14 was observed under a microscope.

\section{Statistical analysis}

Statistical analysis was performed using SPSS 17.0 statistical software analysis. The crystal for- 
mation was compared between groups using the Kruskal-Wallis rank sum test, and the significance level was $\alpha=0.05$.

\section{Results}

\section{Kidney crystal formation in each group}

In the NC group, no crystals were found in the kidney tissue. From the third week, there were bright crystals in the kidneys of the EG group. From the $4^{\text {th }}$ week, the crystals also appeared in the kidneys of the NB group, which were mainly distributed in the distal convoluted tubules and proximal convoluted tubules. Around the glomerulus, the shape of the crystal was irregular. The crystals were scattered and a small amount of crystals were connected to each other into pieces or piles. There was no statistically significant difference in the rate of stone formation between the EG group and the NB group $(p>0.05)$ (Figure 1). There was stone formation from the third week in the EG group and from the fourth week in the NB group. There was also the possibility of stone formation in the rats that had been sacrificed in the first 2 weeks or the first 3 weeks. Therefore, during calculation of the stone formation rate, rats that had been sacrificed in the previous weeks were excluded from the total rat count (Table I).

\section{Immunohistochemistry results of CaSR protein in renal tissues}

Under light microscopy, it was observed that CaSR was continuously expressed in the kidney tissues of the three groups of rats. From the third week, the expression of CaSR protein in renal tissue of the EG group was increased. From the fourth week, the expression of CaSR protein in the kidney tissue of the NB group began to increase, and it was diffusely distributed on the cell membrane of renal tubular epithelial cells, which was brown, while in each week, the expression of CaSR protein in kidney tissue of the EG group was significantly stronger than that of the NB group. From week 1 to 10, CaSR continued to be expressed in the NC group, but its intensity did not change significantly (Figure 2).

\section{Immunohistochemical results of claudin-14} protein in renal tissues of rats

Claudin-14 was continuously expressed in the kidney tissues of the EG group and NB group, but not in the kidney tissue of the NC group. From the third week, the expression of claudin-14 protein in renal tissue of the EG group was increased. From the fourth week, the expression of claudin-14 protein in renal tissue of the NB group also began to increase, and it was diffusely distributed in renal tubular epithelial cells, shown in brown color, while the expression of claudin-14 protein in the EG group was significantly stronger than that in the NB group. From week 1 to 10, claudin-14 continued to be expressed in the NC group, but its intensity did not change significantly (Figure 3).

\section{Stone composition analysis}

In the NC group, 1 (3.3\%) case of calcium oxalate stone and 1 (3.3\%) case of ammonium magnesium phosphate stone were detected, with the total detection rate of $6.7 \%$. In the EG
EG

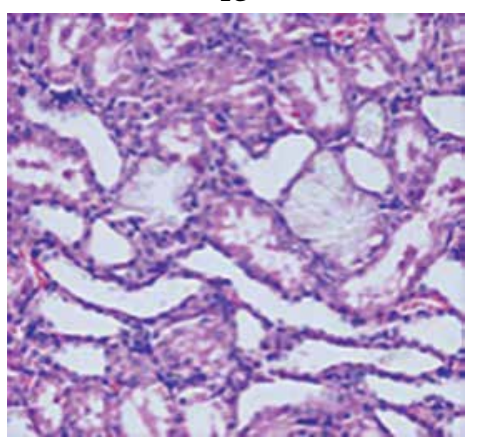

NB

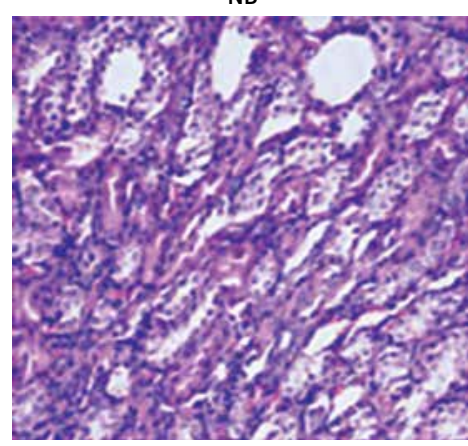

NC

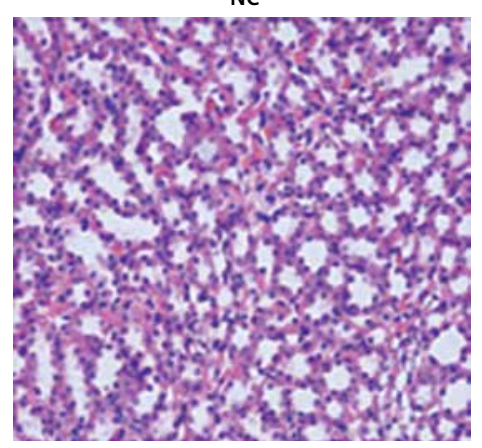

Figure 1. Kidney morphology of each group at eighth week (HE, 200x)

Table I. Renal tissue grading of each group of rats

\begin{tabular}{|cccccccc|}
\hline Group & N & 0 & I & II & III & IV & Stone probability\% \\
\hline EG & 24 & 8 & 9 & 4 & 2 & 1 & 66.7 \\
\hline NB & 21 & 10 & 7 & 3 & 1 & 0 & 52.4 \\
\hline NC & 30 & 30 & 0 & 0 & 0 & 0 & 0 \\
\hline
\end{tabular}

$P=0.792>0.05$ in EG group compared with NB group; $p<0.05$ in EG group compared with NC group; $p<0.05$ in NB group compared with NC group. 
EG

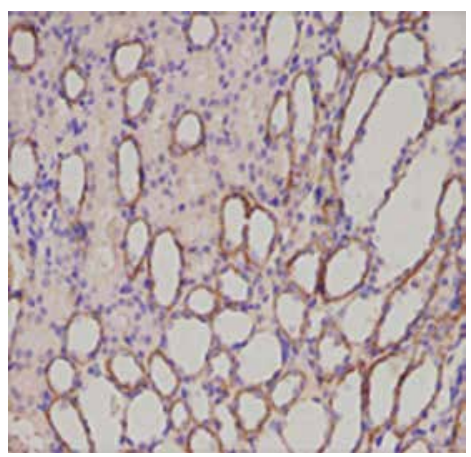

NB

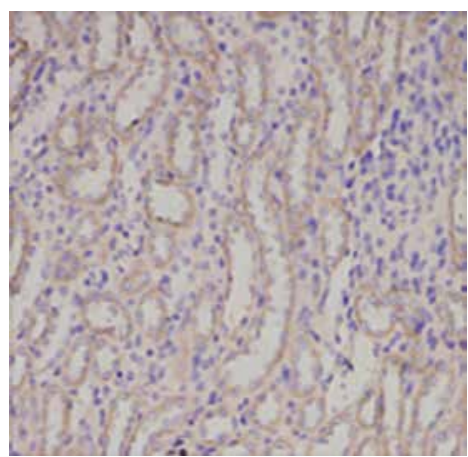

NC

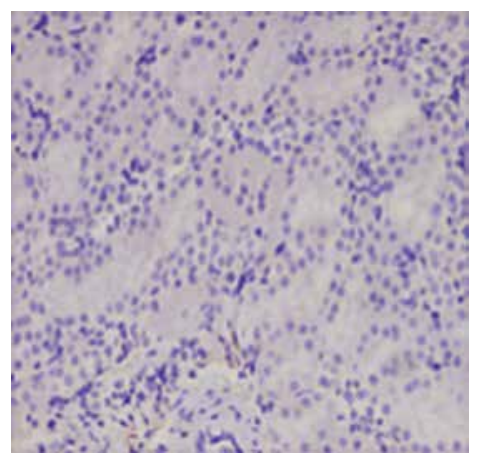

Figure 2. Positive expression of CaSR in kidney tissues of three group at the eighth week (immunohistochemistry, 200x)

EG

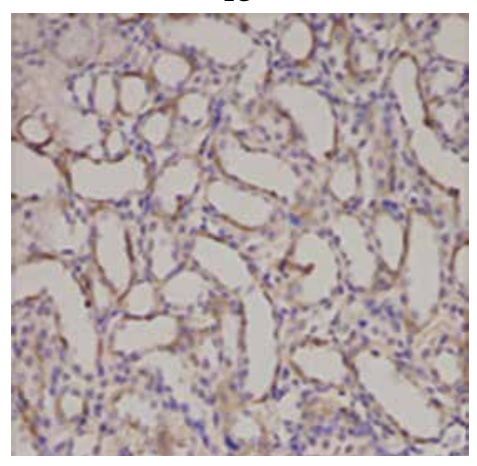

NB

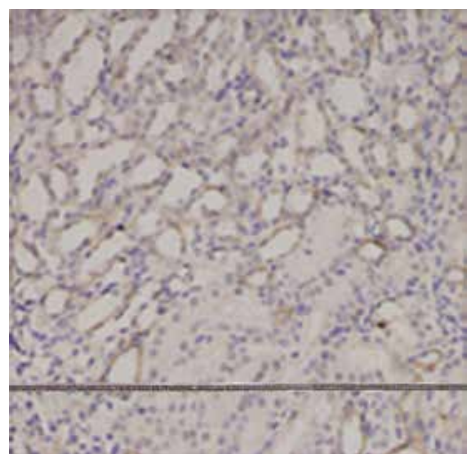

NC

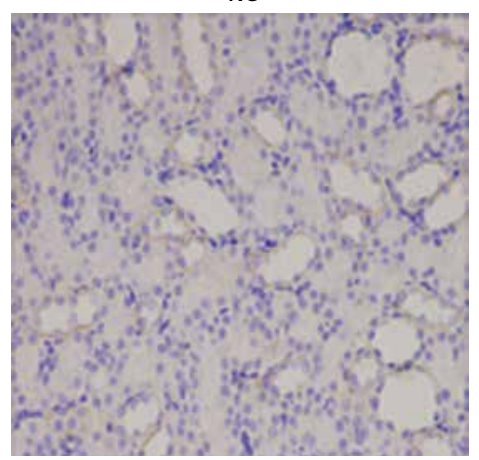

Figure 3. Positive expression of claudin-14 in kidney tissues of three group at the eighth week (immunohistochemistry, 200x)

group, 7 (23.3\%) cases of calcium oxalate stone, $5(16.7 \%)$ cases of calcium oxalate + calcium phosphate stone, $3(10.0 \%)$ cases of calcium oxalate + magnesium ammonium phosphate stone, $1(3.3 \%)$ case of magnesium ammonium phosphate stone, and $3(10.0 \%)$ cases of calcium phosphate stone were detected. The total detection rate was $63.3 \%$. In the NB group, $3(10.0 \%)$ cases of calcium oxalate stones, $3(10.0 \%)$ cases of calcium oxalate + calcium phosphate stones, $2(6.7 \%)$ cases of calcium oxalate + magnesium ammonium phosphate stones, $3(10.0 \%)$ cases of magnesium ammonium phosphate stones, 1 (3.3\%) case of calcium phosphate stone, and $1(3.3 \%)$ case of uric acid stone were detected. The detection rate was $43.3 \%$.

\section{Discussion}

Kidney stones, most of which are calcium oxalate stones, are a common disease. Analysis of the three groups of stones in the present study showed that calcium oxalate-containing stones accounted for $70.6 \%$ of the total number of stones. Many factors can lead to the formation of kidney stones, such as low urine output and high urinary calcium, uric acid, and urine PH. Studies have shown that increased urinary calcium excretion is an important risk factor for inducing or promoting the for- mation of calcium-containing urinary tract stones. The urine is relatively supersaturated to induce the formation of calcium-containing stones [6]. In recent years, with the study of $G$ protein coupled receptors, new treatments are gradually being discovered [7]. CaSR is a G protein-coupled receptor that has been found to be significantly associated with urinary calcium excretion in recent years. It can inhibit the reabsorption of calcium ions in the renal tubules by up-regulating the expression of claudin-14 in the thick ascending $\operatorname{limb}$ (TAL) [4], thereby increasing urinary calcium excretion.

At present, there are no studies on the differences between the two modeling methods of EG and NB. Our group confirmed in the early stage that the increase of urinary calcium in rats with EG-induced kidney stones is related to the CaSR-claudin-14 regulatory pathway [3]. In the past, our research group found that in the process of establishing an NB rat kidney stone model, the $24 \mathrm{~h}$ urinary calcium excretion of NB rats was significantly higher than that of the blank group [8-10]. The role of CaSR and claudin-14 in the NB rat kidney stone model and the EG rat kidney stones and the expression difference between CaSR and claudin-14 is still unclear. Therefore, we established kidney stone models with $\mathrm{EG}$ and NB methods to observe expression of CaSR and claudin-14 protein in kidney tissues of two models. 
In this study, immunohistochemical staining showed that the expression of CaSR and claudin-14 in the EG group gradually increased from the third week; the expression of CaSR and claudin-14 in the NB group increased from the fourth week. There was no increase in expression of CaSR in the kidney tissue of NC rats, and there was no obvious expression of claudin-14 in the kidney tissue of the NC group. The expression of CaSR and claudin-14 in renal tissue of the EG group was significantly higher than that of NB group and NC group. This result may be related to the induction of tubular epithelium in the early stages of modeling with EG and ammonium chloride. Injury [11] promotes the rapid formation of calcium oxalate crystals and increases the concentration of free calcium in urine, thereby activating the CaSR-claudin-14 regulation mechanism and accelerating urinary calcium excretion, while the NB group can form hydroxyapatite stone under physiological conditions. After the formation of this mineralized shell, the NB will be dormant. After invading the body, NB can invade or infect tubular cells through a similar receptor-mediated pinocytosis [12]. EG and ammonium chloride more easily cause damage to renal tubular epithelial cells.

CaSR is widely distributed in various parts of the human kidney, especially the distal convoluted tubule [13], which plays an important role in regulating the excretion of water and electrolytes. It has been found that in distal convoluted tubules, CaSR reduces calcium ion reabsorption and promotes calcium excretion. In the collection tube, water and proton reabsorption can be reduced [14], which can increase the thick section of the upper medullary limb. Claudin-14 inhibits renal tubular reabsorption of calcium ions [4], thereby increasing urinary calcium excretion. The polymorphism of the CaSR gene is closely related to the formation of calcium-containing stones [15]. Tight junction proteins are the most important functional molecules in the body and form tight junctions. Their main functions are to selectively form pores for the penetration of small-sized ions, such as charge-selective pores, and to maintain the unique barrier function and barrier function of tight junctions. Abnormal expression or overexpression can disrupt the structure of epithelial cells and endothelial cells, leading to severe damage to their function. The claudin-14 protein is an important member of the claudin family [16], which regulates the cell-by-cell pathway of tight junction protein-16 (claudin-16) and tight junction protein-19 (claudin-19) The cation channel permeability of the heteropolypeptides is formed to affect the reabsorption of calcium by the renal tubules [17-19]. Dimke et al. [4] found that claudin-14 protein is mainly expressed in the ascending segment of the medullary sputum, inhibiting the reabsorption of calcium and magnesium ions in the thick section of the myelin, thus increasing the urinary calcium concentration, but its inhibition is affected by calcium intake and CaSR, and dysregulation of the renal CaSR-claudin-14 pathway may contribute to the development of significant hypercalciuria, leading to kidney stones and osteoporosis. In this study, CaSR and claudin-14 proteins were strongly expressed in the kidney tissues of two rat kidney stone models, indicating that the CaSR-claudin-14 pathway plays a role in the formation of stones.

In conclusion, the etiology of kidney stones is complex and requires more extensive and in-depth research. In this study, a traditional EG model and an NB model were established, and the stone formation results of the two models and the expression difference of CaSR-claudin-14 were compared. Our results demonstrate that the traditional EG kidney stone modeling method is faster than the NB kidney stone modeling method, with a higher stone formation rate. The expression of CaSR and claudin-14 protein in EG groups was stronger than in NB groups, but the difference was not statistically significant. The NB used to establish the kidney stone model was isolated from patients with kidney stones, which may imitate the process of natural formation of kidney stones of patients. Therefore, the results of our research are more conducive to related research on the etiology of stones.

\section{Acknowledgments}

This study was supported in part by grants from the national Natural Science Foundation of China (no. 81460140).

\section{Conflict of interest}

The authors declare no conflict of interest.

\section{References}

1. Tan J, Chen B, He L, et al. Renal access through the inferior calyx is associated with higher risk of severe bleeding after percutaneous nephrolithotomy. Arch Med Sci 2015; 11: 340-5.

2. Tian Y, Li M, Wu S, et al. Neutrophil elastase stimulates MUC5AC expression in human biliary epithelial cells: a possible pathway of PKC/Nox/ROS. Arch Med Sci 2017; 13: 677-85.

3. Sun W, Wang QZ, Ding GF. Expression and significance of calcium sensitive receptor and tight junction protein-14 in renal tissues of calcium oxalate stone. Tianjin Pharm 2014; 42: 657-60.

4. Dimke H, Desai P, Borovac J. Activation of the Ca2+-sensing receptor increases renal claudin-14 expression and urinary Ca2+ excretion. Am J Physiol Renal Physiol 2013; 304: F761-9.

5. An RH, Feng T, Guo YL. Effects of coumarin on the formation of experimental calcium oxalate stones in rats. Chin J Urol 1994; 15: 209-12. 
6. Wang S. Research progress in polymorphisms associated with high urinary calcium syndrome. Int J Urol 2012; 32: 101-3.

7. Bojic T, Sudar E, Mikhailidis D, et al. The role of G protein coupled receptor kinases in neurocardiovascular pathophysiology. Arch Med Sci 2012; 8: 970-7.

8. Chu H, Wang Q, Wu S, Qian C, Wang Y, Qian B. Dynamic study on the time of stone formation in rat nephrolithiasis model induced bynanobacteria. Chin General Practice 2017; 20: 2613-8.

9. Mo L, Huang HY, Zhu XH. Tamm-Horsfall protein is a critical renal defense factor protecting against calcium ox alate crystal formation. Kidney Int 2004; 66: 1159-66.

10. Shiekh FA, Khullar M, Singh SK. Lithogenesis: induction of renal calcifications by nanobacteria. Urol Res 2006; 34: 53-7.

11. Fassnacht $M$, Libe $R$, Kroiss $M$. Adrenocortical carcinoma a clinician's update. Nat Rev Endocrinol 2011; 7: 323-5.

12. Zheng YB, Wu CT, Huang XC. Progress in nanobacteria research. Chin J Microbiol Immunol 2003; 23: 914-5.

13. Riccardi D. Localization of the extracellular $\mathrm{Ca} 2+$ /polyvalent cation-sensing protein in rat kidney. Am J Physiol 1998; 274: F611-22.

14. Vezzoli G. Calcium-sensing receptor and calcium kidney stones. J Transl Med 2011; 9: 201.

15. Chou YH. A genetic polymorphism (rs17251221) in the calcium-sensing receptor gene (CASR) is associated with stone multiplicity in calcium nephrolithiasis. PLoS One 2011; 6: e25227.

16. Findley MK, Koval M. Regulation and roles for claudin-family tight junction proteins. IUBMB Life 2009; 61: 431-7.

17. Hou J, Renigunta A, Konrad M, et al. Claudin-16 and claudin-19 interact and form a cation-selective tight junction complex. J Clin Investig 2008; 118: 619-28.

18. Konrad M, Schaller A, Seelow D, et al. Mutations in the tight-junction gene claudin 19 (CLDN19) are associated with renal magnesium wasting, renal failure, and severe ocular involvement. Am J Human Genet 2006; 79: 94957.

19. Hou J, Goodenough DA. Claudin-16 and claudin-19 function in the thick ascending limb. Curr Opin Nephrol Hypertens 2010; 19: 483-8. 